\title{
Neurotrophin-3 production promotes human neuroblastoma cell survival by inhibiting TrkC-induced apoptosis
}

\author{
Jimena Bouzas-Rodriguez, ${ }^{1}$ Jorge Ruben Cabrera, ${ }^{1}$ Céline Delloye-Bourgeois, ${ }^{1}$ \\ Gabriel Ichim, ${ }^{1}$ Jean-Guy Delcros, ${ }^{1}$ Marie-Anne Raquin, ${ }^{2}$ Raphaël Rousseau, ${ }^{3}$ \\ Valérie Combaret, ${ }^{3}$ Jean Bénard, ${ }^{4}$ Servane Tauszig-Delamasure, ${ }^{1}$ and Patrick Mehlen ${ }^{1}$ \\ ${ }^{1}$ Apoptosis, Cancer and Development Laboratory-Equipe labellisée "La Ligue," CNRS UMR5238, Université de Lyon, France. \\ ${ }^{2}$ Oncopediatric Department, Gustave Roussy Institute, Villejuif, France. 3INSERM U590-Unité d’Oncologie Moléculaire, \\ Université de Lyon. ${ }^{4}$ Molecular Interactions and Cancer CNRS-UMR 8126, IFR54 Gustave Roussy Institute.
}

\begin{abstract}
Tropomyosin-related kinase receptor $\mathrm{C}(\operatorname{TrkC})$ is a neurotrophin receptor with tyrosine kinase activity that was expected to be oncogenic. However, it has several characteristics of a tumor suppressor: its expression in tumors has often been associated with good prognosis; and it was recently demonstrated to be a dependence receptor, transducing different positive signals in the presence of ligand but inducing apoptosis in the absence of ligand. Here we show that the TrkC ligand neurotrophin-3 (NT-3) is upregulated in a large fraction of aggressive human neuroblastomas (NBs) and that it blocks TrkC-induced apoptosis of human NB cell lines, consistent with the idea that TrkC is a dependence receptor. Functionally, both siRNA knockdown of NT-3 expression and incubation with a TrkC-specific blocking antibody triggered apoptosis in human NB cell lines. Importantly, disruption of the NT-3 autocrine loop in malignant human neuroblasts triggered in vitro NB cell death and inhibited tumor growth and metastasis in both a chick and a mouse xenograft model. Thus, we believe that our data suggest that NT-3/TrkC disruption is a putative alternative targeted therapeutic strategy for the treatment of NB.
\end{abstract}

\section{Introduction}

According to the classic neurotrophic theory, neuronal survival is dependent on neurotrophic factors, such as neurotrophins (1, 2 ). These proteins are crucial to the development of the nervous system, especially as they control the massive developmental loss of excess neurons that fail to adequately connect to their targets. However, over the years, neurotrophins - that include NGF, BDNF, neurotrophin-3 (NT-3), and NT-4/5 (2) - and their tropomyosin-related kinase receptors - TrkA, TrkB, and TrkC - have been shown to play an important role in cancer (reviewed in ref. 3). The overall view is that, as is the case with classic tyrosine kinase receptors, dysregulation of kinase activities through various mechanisms generates survival signals via the PI3K/Akt and Ras/MEK/ MAPK pathways, which in turn promote tumor progression (4).

However, TrkC does not seem to fit this picture: (a) its expression is a good-prognosis factor in different types of cancer and more specifically in melanomas (5), medulloblastomas $(6,7)$ and neuroblastomas (NBs) (8-12); (b) its expression and activation have been shown to trigger apoptosis in medulloblastoma cells (13); and (c) as opposed to TrkA and TrkB, it was recently proposed to act as a dependence receptor (14). Such receptors are able to initiate 2 completely opposite signaling pathways, depending on ligand availability. In the presence of ligand, a positive differentiation, guidance, or survival signal is transduced. In the absence of ligand, however, they induce an active process

Authorship note: Jorge Ruben Cabrera and Céline Delloye-Bourgeois contributed equally to this work. Servane Tauszig-Delamasure and Patrick Mehlen are co-senior authors.

Conflict of interest: The authors have declared that no conflict of interest exists. Citation for this article: J Clin Invest. 2010;120(3):850-858. doi:10.1172/JCI41013. of apoptotic cell death. These dependence receptors also include p75 $5^{\text {ntr }}$, deleted in colorectal cancer (DCC), UNC5H, Patched, and neogenin and the RET, EPHA4, and Alk tyrosine kinase receptors $(15-20)$. The proapoptotic activity of dependence receptors is believed to be important for adequate neuron migration or localization during the development of the nervous system (21-23) but also for inhibiting tumor growth.

Along this latter line, this dependence on ligand presence is also thought to act as a safeguard mechanism to prevent tumor cells from developing in settings of ligand unavailability (reviewed in refs. 24, 25). This view is supported by the fact that in mouse gastrointestinal tracts, disrupting the proapoptotic signaling of a specific dependence receptor by overexpressing its ligand (netrin-1) or inactivating the receptor itself $(\mathrm{UNC} 5 \mathrm{H} 3)$ is associated with intestinal tumor progression $(26,27)$. Thus, a tumor losing a dependence receptor's proapoptotic activity would gain a selective advantage for growth. This can be achieved by 3 possible mechanisms: (a) loss or decrease of expression of the dependence receptor itself as extensively described for DCC (28) and more recently for UNC5H $(25,27),(b)$ inactivation of the death signaling pathway, and (c) autocrine production of the ligand. The latter is of great interest in terms of potential therapy, as extracellular interference between ligand and receptor could drive a tumor cell to apoptosis.

We describe here that a large fraction of aggressive NBs that express TrkC has evolved toward a gain of NT-3 expression. NB, the most frequent solid tumor in young children, is diagnosed as a disseminated (stage 4 ) disease in more than $50 \%$ of cases. NB prognosis is highly variable depending on ploidy, pathology, and MYCN gene amplification (reviewed in ref. 29). Current treatments are often limited and induce deleterious side effects. Therefore, new treatments need to be developed that are more tumor specific, 
A

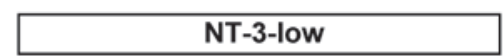

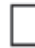
NT-3-high
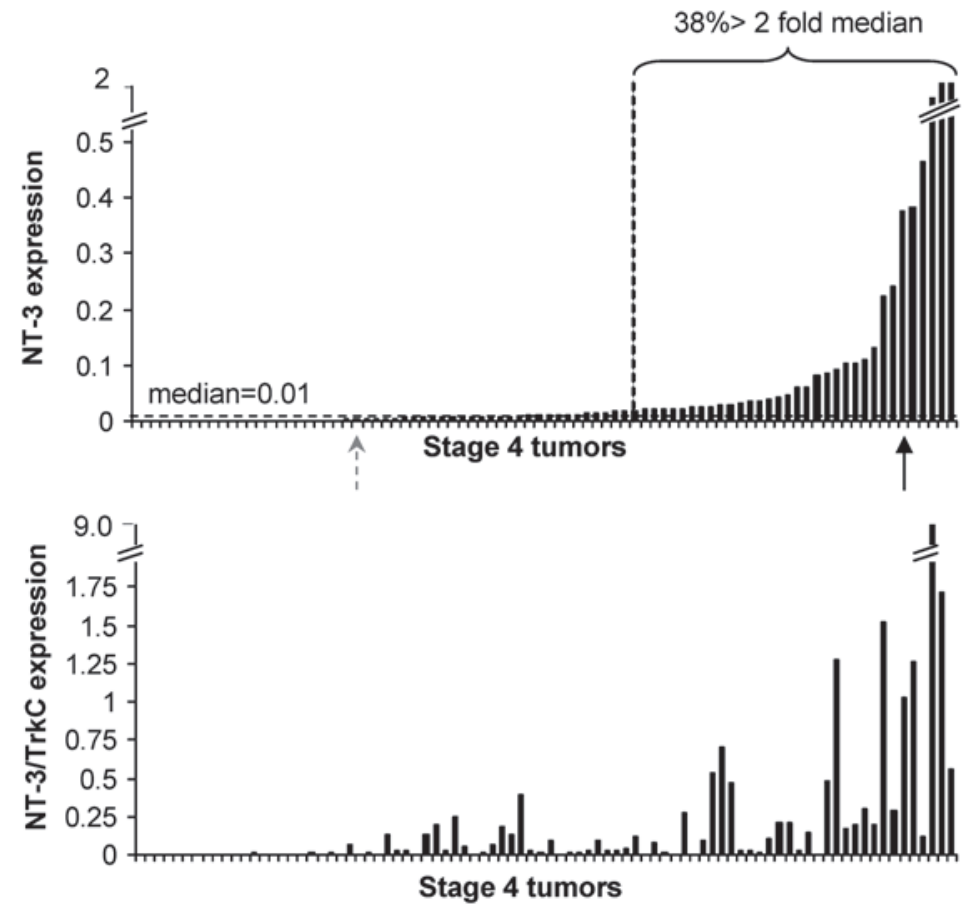

\section{B}

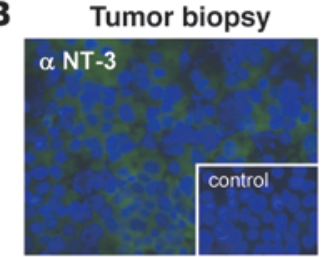

C
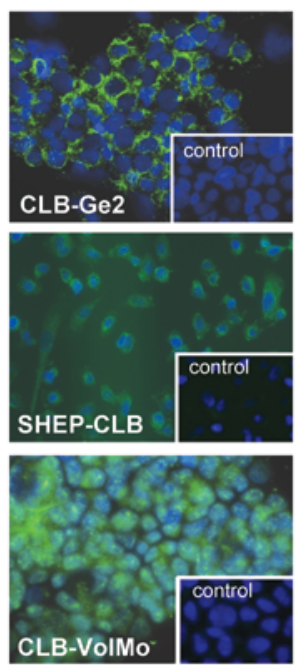

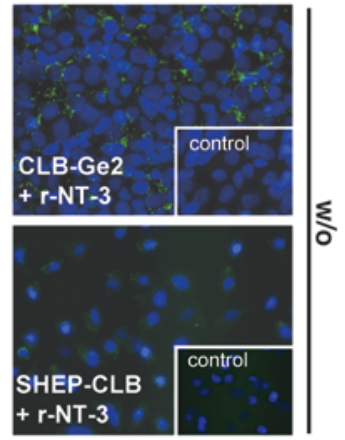

\section{Bone marrow}
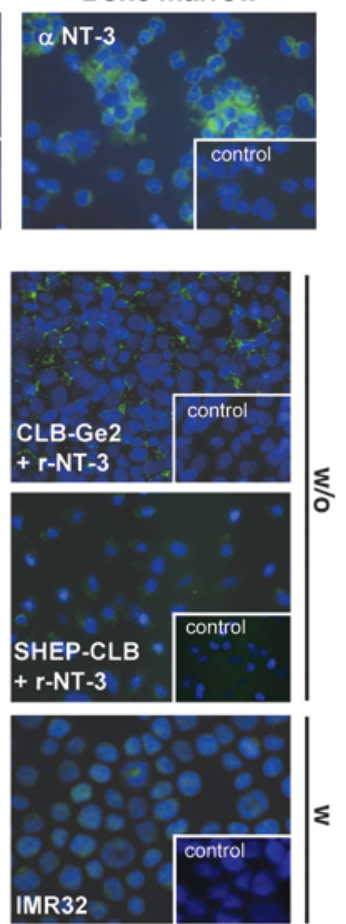

\section{Figure 1}

NT-3 is expressed in a large fraction of stage 4 NBs. (A) NT-3 expression and NT-3/TrkC ratio measured by Q-RT-PCR on total RNA from tumors from a total of 86 stage 4 NB patients. The percentage of tumors expressing NT-3 at more than 2 fold of the value corresponding to the median is indicated. NT-3-low, tumors with low levels of NT-3 expression; NT-3-high, tumors with high levels of NT-3 expression. (B) Representative NT-3 immunohistochemistry on a tumor biopsy and bone marrow dissociated cells from low (left panel) and high (right panel) NT-3-expressing stage 4 patients, corresponding to the dotted gray arrow and black arrow in A, respectively. (C) Representative NT-3 immunohistochemistry on CLBGe2, SHEP-CLB, CLB-VolMo, and IMR32 cells. CLB-Ge2 and SHEP-CLB NT-3 immunostaining, when an excess of recombinant NT-3 ( $r-N T-3)$ is added with primary antibody, is also shown. Note that the 4 top panels show immunohistochemistry performed in absence of membrane permeabilization (w/o), while the immunohistochemistry shown in 2 bottom panels was performed after cell permeabilization with Triton X-100 (w). $(\mathbf{B}$ and $\mathbf{C})$ Insets depict control without primary antibody. Original magnification, $\times 32$.

and consequently, it is key to identify new targets for drug development. We show here that NT-3 expression in NB cells is a selective advantage for tumor cell survival and propose the disruption of the NT-3/TrkC interaction as a novel anticancer strategy to our knowledge in NBs with high NT-3 levels.

\section{Results}

We focused on stage $4 \mathrm{NBs}$, with a specific interest in comparing the expression levels of NT-3 and its receptor TrkC. We first analyzed the expression of NT-3 and TrkC using quantitative RT-PCR (Q-RT-PCR) in a panel of 106 stage 4 NB tumors. A substantial fraction of stage 4 NBs displayed high NT-3 levels (Figure 1A and Supplemental Figure 1A; supplemental material available online with this article; doi:10.1172/JCI41013DS1). Thirty-eight percent of tumors showed at least a 2 -fold increase in NT-3 expression compared with the median value, and more than $20 \%$ of tumors displayed a 5-fold increase (Figure 1A). Tumors with high NT-3 level showed a high NT-3/TrkC ratio, supporting the view of a gain of NT-3 expression in tumors (Figure 1A). We then compared NT-3 levels to the prognosis within the different subcategories of stage $4 \mathrm{NBs}$ - stage 4 either diagnosed before 1 year of age or later and stage $4 \mathrm{~S}$. No significant differences were observed, suggesting that NT-3 upregulation is a selective gain that occurs inde- pendently of tumor aggressiveness and dissemination in a large fraction of stage 4 NBs. Similar results were obtained on stage 1 , 2, or 3 NBs (Supplemental Figure 1A). Expression of NT-3 was not only detected at the mRNA level but also at the protein level by immunohistochemistry (Figure 1B).

NT-3 overexpression was seen in $38 \%$ of stage 4 NBs but also in a fraction of NB cell lines mainly derived from stage $4 \mathrm{NB}$ tumor material (Table 1). Four human NB cell lines, i.e., CLB-Ge2, CLB-VolMo, SHEP-CLB, and IMR32, were studied further. All 4 cell lines express TrkC (data not shown), but CLB-Ge2, CLBVolMo, and SHEP-CLB express high levels of NT-3, whereas NT-3 was barely detected in IMR32 cells both at the messengers level (Table 1) and at the protein level (Figure 1C). Interestingly, NT-3 immunostaining performed on CLB-Ge2 and SHEP-CLB cells, in the absence of cell permeabilization, showed a clear membranous staining. This suggests that the high NT-3 content observed in aggressive NB is associated with an autocrine expression of NT-3 in NB cells. We then started investigating the mechanisms allowing NT-3 a high level of expression in these cells. We failed to detect $N t f 3$ gene amplification or enhanced transcriptional activity of the NT-3 promoter in these cells (data not shown). However, we noticed that inhibition of methyltransferases with the inhibitor 5-aza-2'-deoxycytidine in either CLB-Ge2 or IMR32 cells spe- 


\section{Table 1}

NT-3 expression level and NT-3/TrkC ratio in cell lines derived from NBs

\begin{tabular}{lcc} 
Cell line & NT-3 $\left(\mathbf{1 0} \times \mathbf{1 0}^{-3}\right)$ & NT-3/TrkC \\
Low $\mathbf{N T}-\mathbf{3}$ expression & & \\
IGRN91 & 0.01 & 0.0007 \\
IMR32 & $\mathbf{0 . 0 2}$ & $\mathbf{0 . 0 0 0 0}$ \\
CLB-Es & 0.06 & 0.0002 \\
CLB-BARREC & 0.09 & 0.0007 \\
CLB-BouLT & 0.10 & 0.0017 \\
CLB-Hut & 0.13 & 0.0002 \\
CLB-Ber2 & 0.15 & 0.0007 \\
CLB-Ma2 & 0.15 & 0.0008 \\
SKNAS & 0.18 & 0.024 \\
CLB-Ber1 & 0.20 & 0.0003 \\
CLB-Tra & 0.27 & 0.0016 \\
CLB-Bel & 0.28 & 0.0002 \\
CLB-Re & 0.32 & 0.0010 \\
SHSY 5Y & 0.36 & 0.2887 \\
High NT-3 expression & & \\
CLB-Ma1 & & 0.01 \\
CLB-Bar & 0.57 & 0.04 \\
CLB-Pe & 0.62 & 0.005 \\
SHEP-CLB & 0.62 & $\mathbf{2 . 9}$ \\
CLB-Ga & $\mathbf{0 . 7 4}$ & 0.33 \\
CLB-Sed & 0.75 & 0.006 \\
CLB-Ge1 & 1.02 & 0.01 \\
CLB-Ca & 3.04 & 0.02 \\
CLB-Ge2 & 5.47 & $\mathbf{0 . 1 4}$ \\
CLB-VoIMo & $\mathbf{8 . 0 1}$ & $\mathbf{0 . 0 2}$ \\
\hline NT-3 expren & $\mathbf{9 . 0 4}$ & \\
\hline
\end{tabular}

NT-3 expression and NT-3/TrkC ratio measured by Q-RT-PCR in a fraction of NB cell lines. HPRT expression was used as an internal control. Number of molecules as calculated by $2 \exp (\Delta \mathrm{Cp})$, where $\Delta C p$ is TrkC or NT-3 Cp minus HPRT Cp. Values obtained were divided by 1,000. Bold indicates cell lines further used in this study.

cifically restored NT-3 expression in IMR32 cells (Supplemental Figure 1B). Together with the general hypomethylation associated with cancer, this observation suggests that NT-3 high expression is associated with inhibition of NT-3 promoter methylation.

To investigate whether the NT-3 autocrine expression observed in CLB-Ge2, CLB-VolMo, and SHEP-CLB cells provides a selective advantage for tumor cell survival, as would be expected from the dependence receptor paradigm, cell death was analyzed in response to the disruption of this autocrine loop. As a first approach, NT-3 was downregulated by RNA interference. In this approach, we used either a mix of 3 siRNA sequences (siRNA NT-3) or 2 of them independently (siRNA NT-3-a and siRNA NT-3-b). Efficacy of NT-3 siRNAs tested was evaluated at the Ntf3 mRNA level by Q-RT-PCR (Supplemental Figure 2, A and B) and at the NT-3 protein level by immunohistochemistry (Figure $2 \mathrm{~A}$ ) or by ELISA performed on the conditioning medium (Supplemental Figure 2A). While scrambled siRNA failed to affect cell survival in every cell line studied, as measured by caspase activity (Figure 2B) or ToxiLight (Figure 2C) assays, the transfection of NT-3 siRNAs was associated with CLBGe2, CLB-VolMol, and SHEP-CLB cell death (Figure 2, B and C, and Supplemental Figure 2C). In contrast, IMR32 cell survival was unaffected after NT-3 siRNA treatment (Figure 2, B and C, and Supplemental Figure 2C).
As a second approach, we used a blocking TrkC antibody described before (14) to prevent NT-3 from binding to endogenous TrkC. As shown in Figure 2, D and E, and Supplemental Figure 2D, the addition of anti-TrkC triggered CLB-Ge2 and CLBVolMo apoptotic cell death, as measured by caspase-3 activity assay (Figure 2D) and TUNEL staining (Figure 2E and Supplemental Figure 2D). This effect was specific for NT-3/TrkC inhibition, since the anti-TrkC antibody had no effect on IMR32 cells. Similar CLB-Ge2 cell death induction was observed when, instead of using a blocking TrkC antibody, a recombinant ectodomain of TrkC was used to trap NT-3 (Supplemental Figure 2E).

To determine whether the NB cell death associated with inhibition of the TrkC/NT-3 interaction can be extended to fresh tumors, a surgical biopsy from a tumor and the corresponding bone marrow was semi-dissociated and further incubated with the antiTrkC antibody. This primary tumor and the disseminated neoplasia expressed both NT-3 and TrkC (Supplemental Figure 2F), and an increased cell death, measured by caspase activation, was detected in response to the anti-TrkC antibody (Figure 2F).

There are 2 different paradigms for interpreting cell death associated with the interference of the NT-3/TrkC interaction. According to the classic neurotrophic view, the observed cell death could be a death by "default" that results from the loss of survival signals triggered by the NT-3/TrkC interaction - i.e., MAPK or PI3K pathways activated through TrkC's kinase activity. The dependence receptor method offers a different perspective, more compatible with the fact that TrkC expression is usually a good prognosis factor. In this scenario, blocking the interaction between NT-3 and TrkC leads to unbound TrkC actively triggering apoptosis. As a first approach to discriminate between these 2 possibilities, NB cell death was induced via anti-TrkC antibody treatment, after CLB-Ge2/CLB-VolMo transfection with a dominant-negative mutant for TrkC. This dominant-negative mutant, TrkC-IC D641N, has been shown to specifically inhibit the proapoptotic signaling of unbound TrkC, without affecting its kinase-dependent signaling (14). Expression of the dominantnegative mutant fully blocks anti-TrkC-mediated CLB-Ge2 and CLB-VolMo cell death (Figure 3A and Supplemental Figure 3, $A$ and $B$ ). To further support this observation, we assessed the extent of cell death associated with NT-3 siRNA in settings of downregulation of $\operatorname{TrkC}$ by siRNA. As shown in Figure 3, B and C, and Supplemental Figure 3, C-E, while downregulation of TrkC in all 3 NT-3-expressing cell lines is not associated with cell death as would be expected by the classic loss of survival signaling pathways, this downregulation fully blocks cell death induced by NT-3 siRNA. This supports further the hypothesis that NT-3 upregulation observed in CLB-Ge2, CLB-VolMo, and SHEP-CLB cells inhibits the proapoptotic signaling triggered by TrkC itself. Along this line, addition of the blocking TrkC antibody to CLB$\mathrm{Ge} 2$ failed to be associated with a decrease in the classic survival pathways, as exemplified here by measurement of ERK or Akt phosphorylation (Figure 3D). Moreover, TrkC caspase cleavage was enhanced by the TrkC blocking antibody (Figure 3E). Indeed, as previously described, $\operatorname{TrkC}$ and dependence receptors in general are cleaved by caspase, and this cleavage is a prerequisite for their proapoptotic activity $(14,16)$. As shown in Figure 3E, while a basal level of TrkC cleavage was detected in control conditions, addition of the blocking antibody was associated with increased TrkC cleavage, a cleavage blocked by addition of the general and potent caspase inhibitor, Boc-Asp(Ome) fluoromethyl 
A

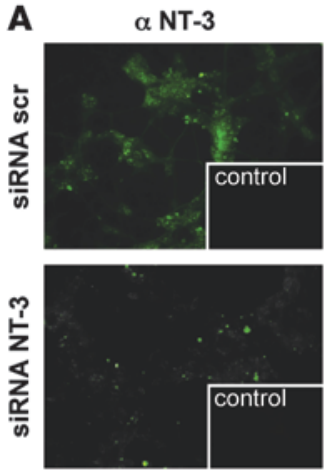

D

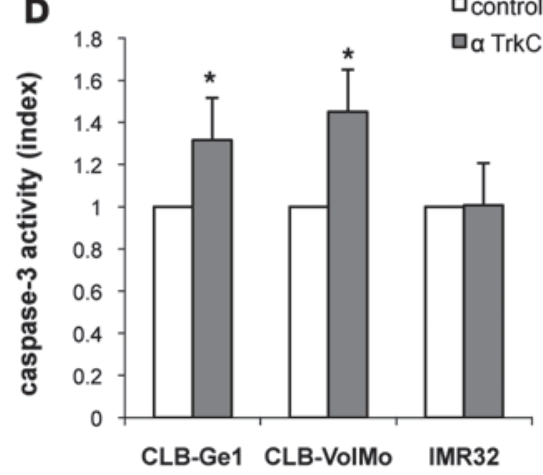

Hoechst $/ \alpha$ NT-3
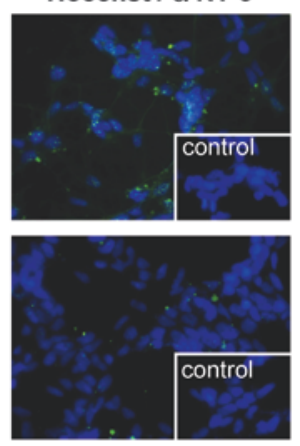

$\square$ control

a TrkC

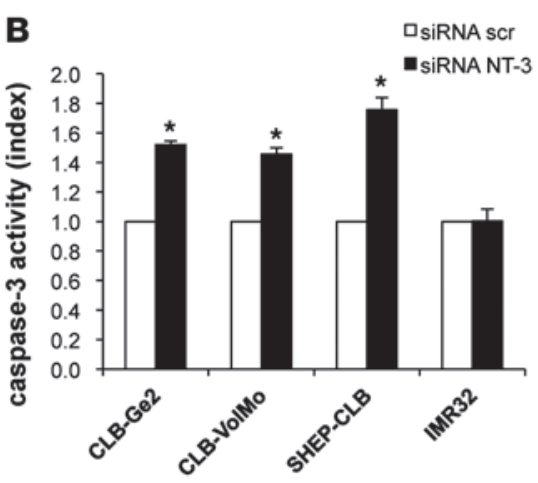

E

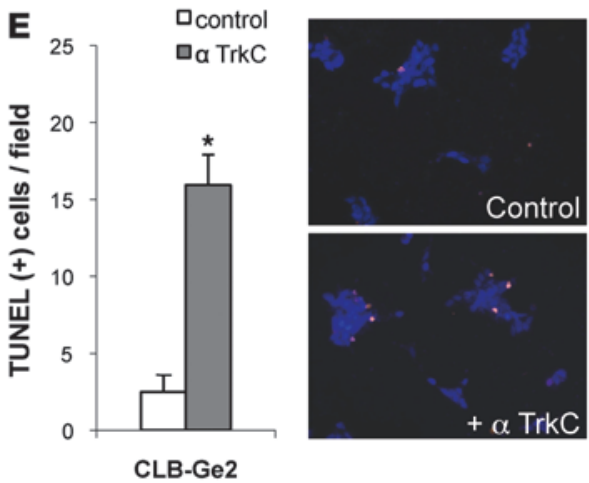

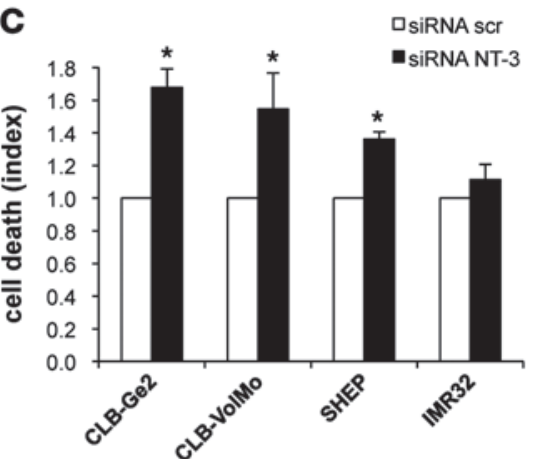

F

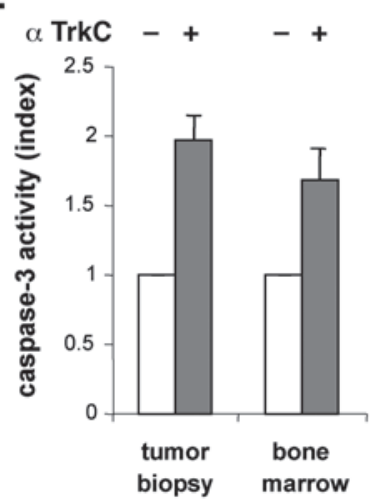

Figure 2

Disruption of NT-3 autocrine loop triggers NB cell death. (A) NT-3 immunostaining on the CLB-Ge2 cell line 24 hours after transfection with scrambled siRNA (siRNA scr) or with NT-3 siRNA (siRNA NT-3). Insets depict control without primary antibody. Original magnification, $\times 32$. (B and C) Cell death induction in CLB-Ge2, CLB-VolMo, SHEP-CLB, and IMR32 cell lines was quantified after transfection with either scrambled siRNA or a mix of 3 siRNAs targeting NT-3, using relative caspase-3 activity assay (B) or Toxilight assay (C). (D and E) Cell death induction in CLB-Ge2, CLB-VolMo, or IMR32 cell lines was quantified in cells treated with anti-TrkC blocking antibody ( $\alpha$ TrkC) or without (control) anti-TrkC antibody, using relative caspase-3 activity assay (D) or TUNEL assay (E). For the TUNEL assay, a representative labeling of TUNEL-positive cells is shown (top panel, control cells; bottom panel, cells treated with anti-TrkC blocking antibody). Original magnification, $\times 20$. (F) Effect of antiTrkC blocking antibody on stage 4 NB. Tumoral cells were directly dissociated from the surgical biopsy and were plated for 24 hours in presence $(+)$ or in absence $(-)$ of treatment. (B-F) Data represent mean \pm SEM. ${ }^{*} P<0.05,2$-sided Mann-Whitney test, compared with control.

ketone (BAF). Together, these data demonstrate that NT-3 upregulation observed in NB cells inhibits the proapoptotic signaling triggered by the dependence receptor TrkC.

We next assessed whether in vivo interference with NT-3/TrkC could be used to limit/inhibit NB progression and dissemination. A chicken model was developed in which grafts of NB cells in the chorioallantoic membrane (CAM) of 10-day-old chick embryos recapitulate both tumor growth at a primary site, within the CAM, as well as tumor invasion and dissemination at a secondary site, metastasis to the lung (ref. 30 and Figure 4A). In a first approach, CLB-Ge2 or IMR32 cells were loaded in 10-day-old CAM, and embryos were treated on day 11 and day 14 with anti-TrkC or an unrelated antibody. Seventeen-day-old chicks were then analyzed for primary tumor growth and metastasis to the lung. As shown in Figure 4, B and D, treatment with the anti-TrkC antibody significantly reduced primary tumor size, specifically in CLB-Ge2-grafted CAM, while an unrelated isotopic antibody had no effect. This size reduction was associated with increased tumor cell apoptosis, as shown by an increased TUNEL staining in the tumors treated with anti-TrkC (Figure 4C). More importantly, anti-TrkC also reduced lung metastasis formation in CLB-Ge2-grafted embryos (but not in IMR32-grafted embryos), as shown in Figure 4E.
To more specifically address whether the anticancer effect of the TrkC blocking antibody was due to the reengagement of TrkCinduced apoptosis through NT-3/TrkC interaction inhibition, we first analyzed the effect of NT-3 siRNA treatment on primary tumors in the chicken model. CLB-Ge2 cells were loaded on a 10-day-old CAM. Embryos were treated, through injection in the CAM blood vessels, on day 11 and day 14 with a siRNA targeting NT-3 or scrambled siRNA. Seventeen-day-old chicks were then analyzed for primary tumor growth. As shown in Figure 4F, NT-3 siRNA treatment was associated with a significant antitumor effect. Of interest, when TrkC siRNA treatment was assessed instead of NT-3 siRNA, no effect on tumor size was observed, further suggesting that the antitumor effect seen with the blocking antibody or with NT-3 siRNA is not associated with a "classic" loss of the survival signals promoted by the tyrosine kinase activity of TrkC. On the other hand, we show that a dual treatment with NT-3 siRNA and TrkC siRNA reversed the anticancer effect observed in NT-3 siRNA-treated CAM (Figure 4F). This result thus demonstrates that NT-3 inhibition is associated with tumor growth inhibition and cell death, because it reengages TrkC-induced apoptosis.

To further provide a proof of concept that NT-3 interference may represent a putative alternative therapeutic approach, classic xeno- 
A

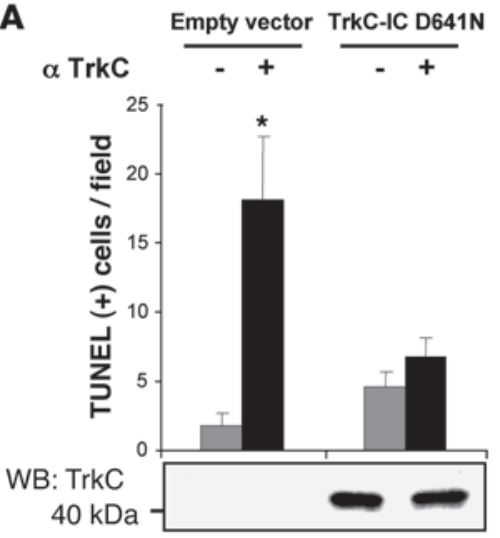

C

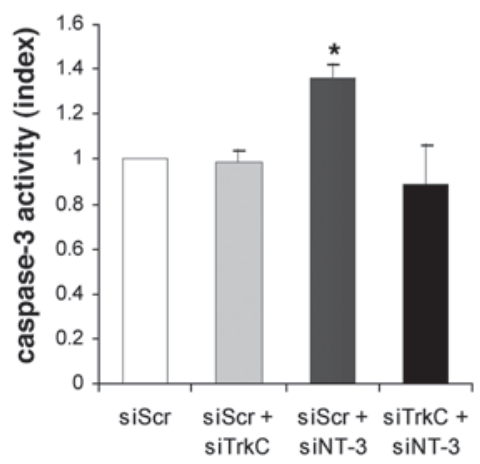

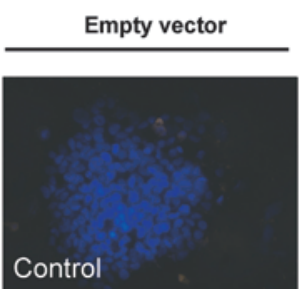

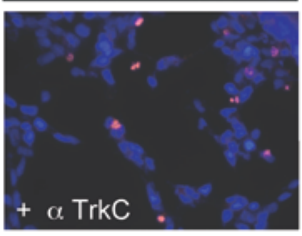

Control

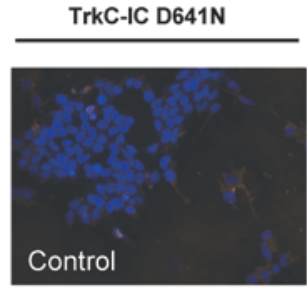

D

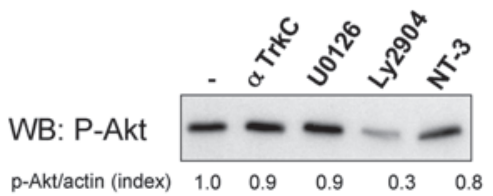

WB: P-Erk

p-Erk/actin (index)

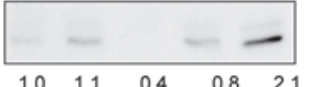

WB: $\beta$-actin
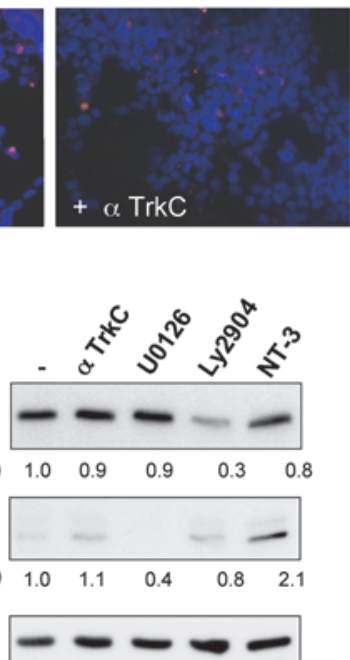
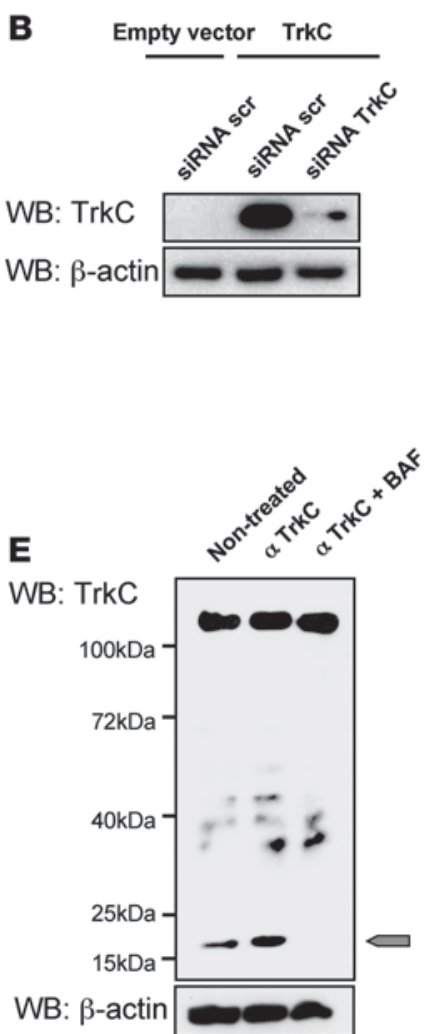

Figure 3

NT-3/TrkC interference promotes TrkC proapoptotic activity. (A) CLB-Ge2 cells were transfected with either empty vector or with a plasmid encoding the dominant-negative TrkC-IC D641N and treated for 24 hours with or without anti-TrkC blocking antibody Cell death was monitored by TUNEL labeling of cells plated on slides. The control panel shows TrkC-IC D641N by Western blots using anti-TrkC antibody (bottom panel). Representative images are shown. Original magnification, $\times 20$. (B) The efficacy of TrkC siRNA was evaluated by Western blot on nonexpressing TrkC 13.S.24 olfactive neuroblasts. Cells were transfected either with empty vector or with uncleavable TrkC D945N D641N double mutant that does not trigger apoptosis, and with scrambled siRNA or TrkC siRNA (siRNA TrkC). (C) Cell death induction in the CLB-Ge2 cell line was quantified after transfection with either scrambled siRNA (siScr), TrkC siRNA (siTrkC), NT-3 siRNA (siNT-3), or a mix of TrkC and NT-3 siRNA, using relative caspase-3 activity assay. (D) Phospho-Akt and phospho-Erk levels of CLB-Ge2 cells were monitored by Western blot after 16

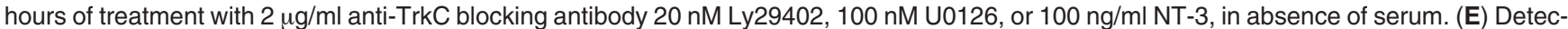
tion of TrkC cleavage band ( $20 \mathrm{kDa}$, indicated by the arrow) by Western blot, using an anti-TrkC antibody on cells treated (or not) with anti-TrkC blocking antibody, with or without the general caspase inhibitor BAF. (A and C) Data represent mean \pm SEM. ${ }^{*} P<0.05,2$-sided Mann-Whitney test, compared with control.

grafts of NB cells in nude mice were performed. SHEP-CLB were xenografted subcutaneously in nude mice. When the engrafted tumors reached $100 \mathrm{~mm}^{3}$, mice were treated intraperitoneally 3 time a week, either with NT-3 siRNA or with scrambled siRNA as successfully performed previously $(31,32)$. As shown in Figure 5, the growth of tumors engrafted in mice treated with NT-3 siRNA was strongly reduced compared with the ones engrafted in scrambled siRNAtreated mice. This tumor growth inhibition was associated with a reduction in tumor weight. Together, these data further support the antitumor effect associated with NT-3 interference in vivo.

\section{Discussion}

We show here that a fraction of NBs show an autocrine production of NT-3 associated with an increased NT-3/TrkC ratio. This elevated NT-3/TrkC ratio likely confers a selective advantage acquired by the cancer cells in settings of limited/no NT-3. Interestingly, this dependence on NT-3 presence appears specific for TrkC and does not involve other Trk receptors (i.e., TrkA or TrkB), as a dominant negative of TrkC is sufficient to turn down this dependence (Figure 3A).
Both NT-3 and TrkC have been shown to be expressed in various cancers $(5,33-47)$. However, most of the field has concentrated its attention on the expression of $\operatorname{TrkC}$, considered as a favorable outcome factor in NB and medulloblastoma (6-8, 10-12). All these studies view NT-3 as an accessory compound that complements TrkC autoactivation and induces proliferation through TrkC kinase-dependent signaling. Along this line, NT-3 was shown to induce survival and differentiation of primary cultures derived from NB (48) and further to activate heparanase activity through TrkC and be involved in invasion during metastasis (49-51).

Here, we show that autocrine NT-3 expression is a mechanism developed by a large fraction of tumor cells to bypass TrkCinduced cell death that would occur in regions of limited NT-3 concentrations. This selective advantage is, however, only 1 of 3 possible means a tumor can use to inactivate TrkC-induced apoptosis, the 2 others being either a loss of TrkC expression or an inactivation of the downstream proapoptotic signaling. Along this later line, IMR32 cells are NT-3-negative cells and undergo apoptosis through forced expression of Bax, a classic cell death inducer. 
A

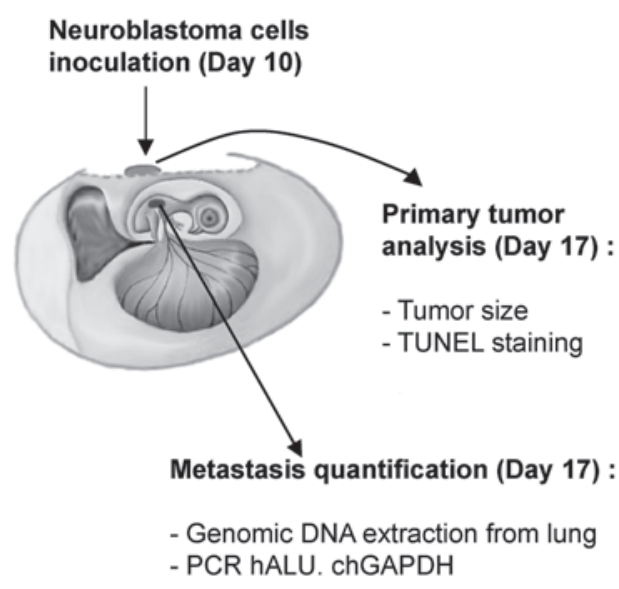

B
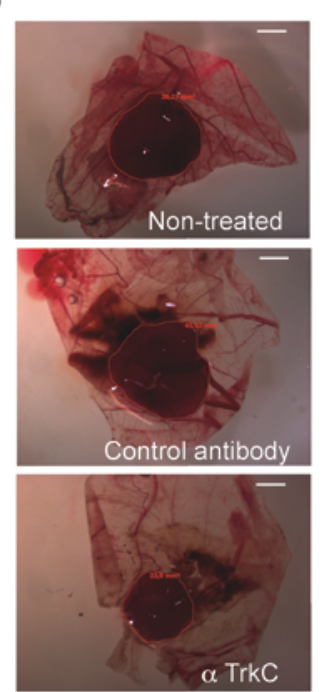

C TUNEL
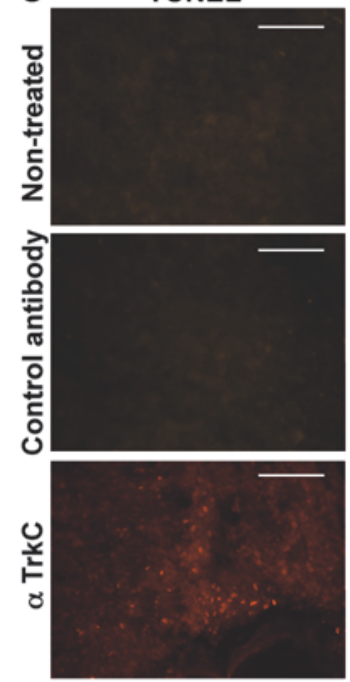

TUNEL / Hoechst
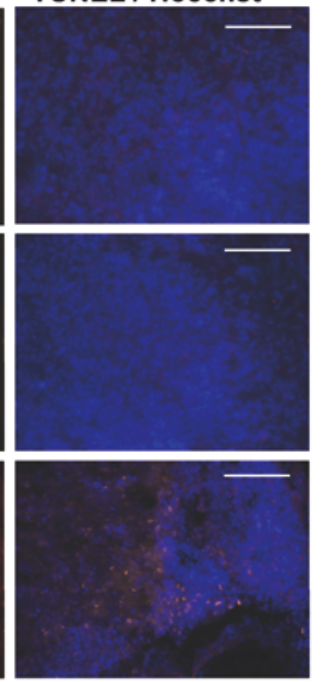

$\mathbf{F}$
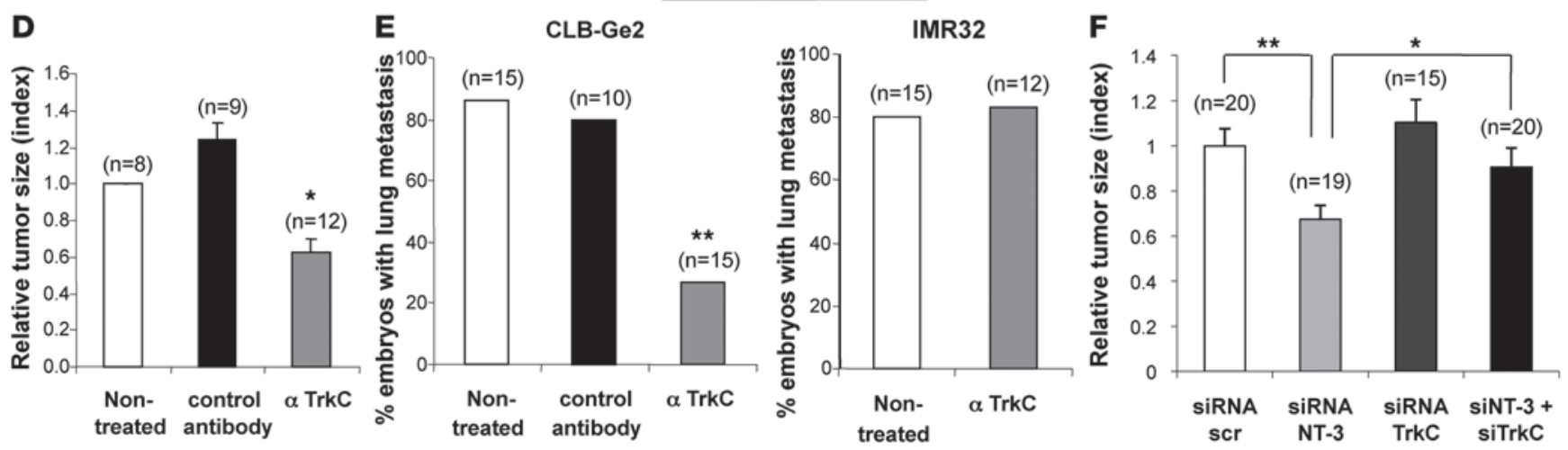

Figure 4

Blocking NT-3/TrkC inhibits NB growth and dissemination. (A) Schematic representation of the experimental chick model. IMR32 or CLB-Ge2 cells were grafted in CAM at day 10, and anti-TrkC blocking antibody or an isotypic antibody (control antibody) was added on day 11 and day 14 . Tumors and lungs were harvested on day 17. (B-D) Effect of anti-TrkC blocking antibody on primary tumor growth and apoptosis. (B) Representative images of CLB-Ge2 primary tumors formed on nontreated CAM or CAM treated either with an isotypic antibody (control antibody) or with anti-TrkC blocking antibody. Scale bar: $2 \mu \mathrm{m}$. (C) Representative images of TUNEL-positive cells in the respective primary tumors described in B. Scale bar: $100 \mu \mathrm{m}$. (D) Quantitative analysis showing the primary tumor size relative to nontreated tumors. (E) Effect of anti-TrkC blocking antibody on lung metastasis. The percentage of embryos with lungs invaded by IMR32 or CLB-Ge2 cells after 2 intratumoral injections (day 11 and day 14) of either anti-TrkC blocking antibody, an isotypic antibody, or nontreated. (F) Same as for D except that NT-3, TrkC, scrambled, or NT-3 and TrkC siRNA were injected on a chorioallantoic vessel. Relative primary tumor size is presented. (D and F) Data represent mean \pm SEM. ${ }^{\star} P<0.05,{ }^{\star \star} P<0.01,2$-sided Mann-Whitney test, compared with nontreated tumors. (E) ${ }^{\star \star} P<0.01, \chi^{2}$ test.

Nevertheless, they fail to die after TrkC overexpression (Supplemental Figure 4), thus suggesting that in IMR32 cells, the loss of TrkC proapoptotic signaling has been selected. As a consequence, NT-3 high expression constitutes a new marker for NB patients that could putatively respond to a treatment based on cell death induction via disruption of the NT-3/TrkC interaction. One may wonder, what are the mechanisms leading to NT-3 upregulation. Our preliminary results suggest that NT-3 expression in cancer cells may be regulated by epigenetic mechanisms, but further work will be needed to address this important point.

The in vitro cell death effect and the in vivo antitumor effect of NT-3 interference on NT-3-expressing tumor cells support the view that NT-3 is an exciting target for drug development. So far, the data obtained are still limited in term of the number of models tested and also on the amplitude of the anticancer effect observed (Figures 4 and 5). This, however, could be explained by the fact that the tools used here (siRNA or polyclonal antibody) were proof-ofconcept tools and definitely not drug candidate tools. Drug development has now to be explored. However, our data calls for a larger screen of cancers that could be responsive to such a therapeutic approach. Indeed, the therapeutic strategy presented here is fundamentally different from the now classic strategies for cancer therapy based on inhibitors of tyrosine kinase activity. In our view, inhibiting the kinase activity-dependent survival signals triggered by TrkC should only have poor tumor death effect, i.e., cytostatic, if the drug developed does not also target the activation of the proapoptotic activity of TrkC. We propose here that a treatment based on inhibition of the interaction between NT-3 and its dependence receptor TrkC, by blocking either NT-3 or TrkC, could potentially provide benefit, as a first line treatment or in combination with standard chemotherapy, to the large fraction of the patients suffering from aggressive NB and possibly other cancers with high NT-3 levels. 
A

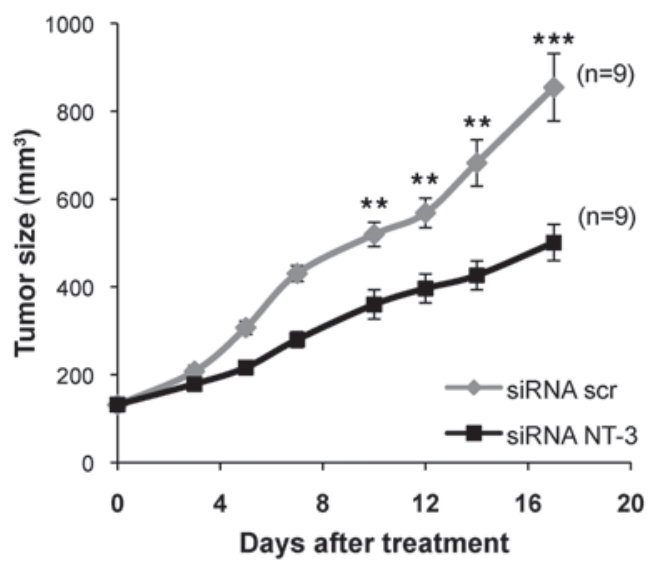

B

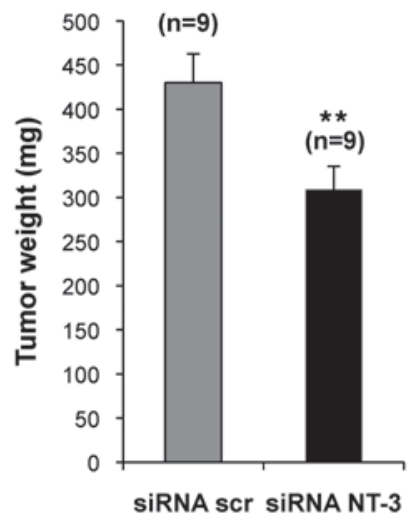

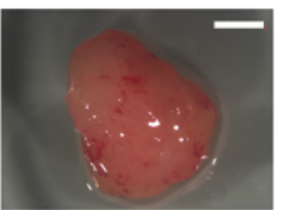
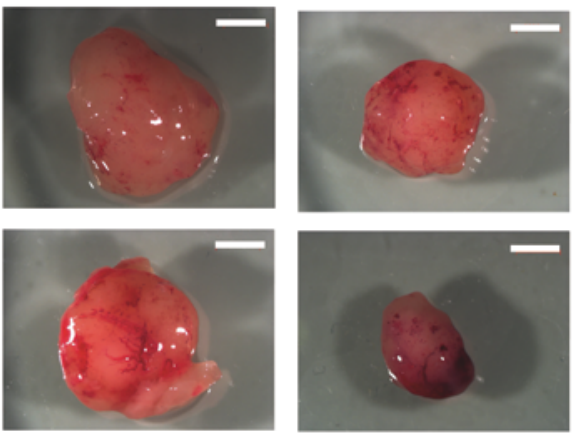

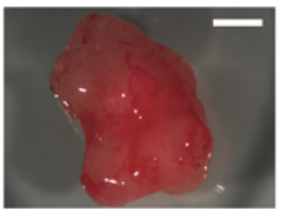

siRNA scr

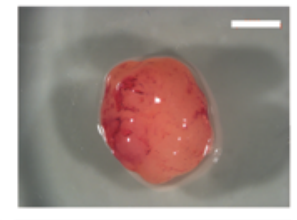

SIRNA NT-3

Figure 5

Disruption of NT-3 autocrine loop inhibits NB tumor growth. (A) The volume of SHEP-CLB engrafted tumors was measured during treatment with either NT-3 siRNA or scrambled siRNA. Mice were injected intraperitoneally with $3 \mu \mathrm{g}$ siRNA 3 times per week. Mean tumor volume is indicated. (B) SHEP-CLB tumors were dissected after 18 days of treatment. Final tumor weight and representative pictures are shown. Scale bar: $2 \mathrm{~mm}$. Data represent mean \pm SEM. ${ }^{* *} P<0.01,{ }^{* * *} P<0.001,2$-sided Mann-Whitney test, compared with scrambled siRNA-treated mice.

\section{Methods}

Human NB and breast tumors samples and biological annotations. After the consent of parents, surgical human NB tumor material was immediately frozen. Materials and annotations were obtained from the Biological Resources Centers of both national referent institutions for NB treatment, i.e., Centre Léon Bérard and Institut Gustave Roussy. Approval of care and use of these samples was given by the Biological Resources Center Ethical and Steering committees of Centre Léon Bérard and Institut Gustave Roussy.

Cell lines, transfection procedure, and reagents. Human NB cell lines were from the tumor banks at Centre Léon Bérard and at Institut Gustave Roussy. CLB-Ge2, CLB-VolMo, SHEP-CLB, and IMR32 cell lines were cultured in RPMI 1640 Glutamax medium (Gibco) containing 10\% fetal bovine serum. Cells were transfected using Lipofectamine 2000 Reagent (Invitrogen). Tumor biopsies and bone marrow cells were immediately dissociated and cultured on RPMI 1640 Glutamax medium (Gibco) containing 10\% fetal bovine serum. Olfactory neuroblasts 13.S.24 were cultured and transfected as previously described (14). Anti-TrkC blocking antibody was obtained from R\&D Systems (catalog AF1404). The recombinant TrkC/Fc chimera corresponding to the extracellular domain of human TrkC (Fc-TrkC-EC) was obtained from R\&D Systems (catalog 373-TC). BAF caspase inhibitor $(20 \mathrm{mM})$ and 5-aza-2'-deoxycytidine methylation inhibitor (5 $\mu \mathrm{M})$ were from Sigma-Aldrich (A3656).

Plasmid constructs and siRNA. The TrkC dominant-negative mutant TrkC-IC D641N and uncleavable TrkC D495N/D641N were described before (14). Scrambled siRNA (catalog sc-37007) and NT-3 siRNA (catalog sc-42125, sc-42125-A, and sc-42125-B) were obtained from Santa Cruz Biotechnology Inc. TrkC siRNA was from Sigma-Aldrich (SASI_Hs01_00192145 and SASI_Hs01_00192145_AS).

Cell death assays. $2 \times 10^{5}$ cells were grown in serum-poor medium and were treated (or not) with $2 \mu \mathrm{g} / \mathrm{ml}$ anti-TrkC antibody (catalog AF1404, R\&D Systems) and $2 \mu \mathrm{g} / \mathrm{ml} \mathrm{Fc-TrkC-EC} \mathrm{(catalog} \mathrm{373-TC;} \mathrm{R \& D} \mathrm{Systems)}$ or transfected with siRNA or TrkC constructs, using Lipofectamine 2000 (Invitrogen) for CLB-Ge2, CLB-VolMo, and SHEP-CLB cells or Lipo- fectamine Plus for IMR32 cells (Invitrogen). Cell death was analyzed 24 hours after treatment/transfection either by trypan blue exclusion as described previously (15) or with ToxiLight Bio Assay kit (Lonza). Apoptosis was monitored by measuring caspase- 3 activity as described previously (15), using the ApoAlert CPP32 Kit from Clontech (USA). For detection of DNA fragmentation, CLB-Ge2 cells were grown in poly-L-lysine-coated slides and fixed with 4\% PFA 24 hours after treatment/transfection. IMR32- and CLB-VolMo-transfected cells were cytospun before PFA fixation. TUNEL was performed with $300 \mathrm{U} / \mathrm{ml}$ TUNEL enzyme and $6 \mu \mathrm{M}$ biotinylated dUTP (Roche Diagnostics), as described previously (52).

Q-RT-PCR. To assay NT-3 and TrkC expression in NB samples, total RNA was extracted from histologically qualified tumor biopsies (> $60 \%$ immature neuroblasts), using the Nucleospin RNAII Kit (Macherey-Nagel), and 200 ng were reverse transcribed using 1 U SuperScript II Reverse Transcriptase (Invitrogen), 1 U RNAse inhibitor (Roche Applied Science), and $250 \mathrm{ng}$ random hexamer (Roche Applied Science). Total RNA was extracted from human cell lines using the Nucleospin RNAII Kit (Macherey-Nagel), and $1 \mu \mathrm{g}$ was reverse-transcribed using the iScript cDNA Synthesis Kit (BioRad). Real-time Q-RT-PCR was performed on a LightCycler 2.0 apparatus (Roche), using the Light Cycler FastStart DNA Master SYBER Green I Kit (Roche). Q-RT-PCR was performed using the following primers: TrkC, forward 5'-AGCTCAACAGCCAGAACCTC-3' and reverse 5'-AACAGCGTTGTCACCCTCTC-3'; NT-3, forward 5'-GAAACGCGATGTAAGGAAGC$3^{\prime}$ and reverse 5'-CCAGCCCACGAGTTTATTGT-3'. The ubiquitously expressed human HPRT gene, showing the least variable expression in NB, was used as an internal control, using the following primers: forward 5'-TGACACTGGCAAAACAATGCA-3' and reverse 5'-GGTCCTTTTCACCAGCAAGCT- $3^{\prime}$. For all 3 couples of primers, polymerase was activated at $95^{\circ} \mathrm{C}$ for 10 minutes, followed by 35 cycles at $95^{\circ} \mathrm{C}$ for 10 seconds, $60^{\circ} \mathrm{C}$ for 10 seconds, and $72^{\circ} \mathrm{C}$ for 5 seconds.

Immunohistochemistry and immunoblot. $8 \times 10^{4}$ cells were cytospun on coverslips and fixed in 4\% PFA. The slides were then incubated at room temperature for 1 hour with an antibody recognizing human NT-3 (1:300; 
catalog sc-547, Santa Cruz Biotechnology Inc.). After rinsing in PBS, the slides were incubated with an Alexa Fluor 488-conjugated donkey antirabbit antibody (Molecular Probes). Nuclei were visualized with Hoechst staining (Sigma-Aldrich).

Expression of TrkC constructs and endogenous TrkC cleavage were monitored by Western blot with anti-Trk antibody (catalog sc-11; Santa Cruz Biotechnology Inc.), and an anti $\alpha$-actin (13E5; Cell Signaling Technology) was used as loading control as previously described (14).

Phospho-Akt and phospho-Erk levels of CLB-Ge2 cells were measured by Western blot with anti-phospho-Akt (4058, Cell Signaling Technology) and phospho-Erk1 and Erk2 (E7028, Sigma-Aldrich) after 16 hours of culture on serum-free medium with $2 \mu \mathrm{g} / \mathrm{ml}$ anti-TrkC antibody (catalog AF1404, R\&D Systems), 20 nM Ly29402 (Sigma-Aldrich), 100 nM U0126 (Sigma-Aldrich), or $100 \mathrm{ng} / \mathrm{ml} \mathrm{NT-3} \mathrm{(Abcys).}$

ELISA determination of NT-3 level. For NT-3 determination in cell culture supernatants, 13.S.24 cells were transfected with a human NT-3 construct and scrambled siRNA or NT-3 siRNA. Supernatants were collected 24 hours later. A white 96-well plate (CORNING) was coated with a monoclonal human NT-3 antibody (MAB267, R\&D systems), blocked with bovine serum albumin, and incubated with the samples, followed by detection of NT-3 by using a biotinylated anti-human NT-3 antibody (BAF267, R\&D systems), a streptavidin-peroxidase polymer (S2438, Sigma-Aldrich), and a chemiluminescent substrate (Pierce ECL Western Blotting Substrate). The luminescence was read on a Tecan Infinite P500 luminometer. Human recombinant NT-3 (P450-03, Abcys) was used as standard in the ELISA.

Chicken model for NB progression and dissemination. $10^{7} \mathrm{NB}$ cells suspended in $40 \mu \mathrm{l}$ complete medium were seeded on 10-day-old (day 10) chick CAM. $2 \mu \mathrm{g}$ anti-TrkC antibody or an isotypic unrelated antibody (catalog sc-1290, Santa Cruz Biotechnology Inc.) were injected in the tumor on day 11 and day 14. For siRNA treatment, $3 \mu \mathrm{g}$ of scrambled, TrkC, or NT-3 siRNA were injected in a chorioallantoic vessel on days 11 and 14 . On day 17, tumors were resected, and area was measured with AxioVision Release 4.6 software (Zeiss). To monitor apoptosis on primary tumors, they were fixed on $4 \%$ PFA, cryoprotected by overnight treatment with $30 \%$ sucrose, and embedded in Cryomount (Histolab). TUNEL staining was performed on tumor cryostat sections (Roche Diagnostics), and nuclei were stained with Hoechst. To assess metastasis, lungs were harvested from the tumor-bearing embryos and genomic DNA was extracted with the NucleoSpin Tissue Kit (Macherey Nagel). Metastasis was quantified by Q-RT-PCR detection of the human Alu sequence, using the following primers: forward 5'-ACGCCTGTAATCCCAGCACTT-3' and reverse 5'-TCGCCCAGGCTGGAGTGCA-3'. Chick GAPDH-specific primers were used as controls, using the following primers: forward 5'-GAGGAAAGGTCGCCTGGTGGATCG-3' and reverse 5'-GGTGAGGACAAGCAGTGAGGAACG-3'. For both couples of primers, amplification was assessed at $95^{\circ} \mathrm{C}$ for 2 minutes, followed by 30 cycles at $95^{\circ} \mathrm{C}$ for 30 seconds, $63^{\circ} \mathrm{C}$ for 30 seconds, and $72^{\circ} \mathrm{C}$ for 30 seconds. Genomic DNA extracted from lungs of noninoculated chick embryos was used to determine the threshold.

Xenograft of human NB cells in nude mice. Five-week-old (20-22 g body weight) female athymic $n u / n u$ mice were obtained from Charles River. Approval of care and use of mice was given by ethical committees of Centre Léon Bérard and by the French Ministry of Research. The mice were housed in sterilized filter-topped cages and maintained in a pathogen-free animal facility. SHEP-CLB cells were implanted by subcutaneous injection of $5 \times 10^{6}$ cells in $200 \mu \mathrm{l}$ PBS into the flank of the mice to make 1 tumor per mouse. When tumors reached a volume of $100 \mathrm{~mm}^{3}$, approximately 11 days after injection, $3 \mu \mathrm{g}$ of either scrambled or NT-3 siRNA, diluted in $300 \mu \mathrm{l}$ of PBS, were injected intraperitoneally 3 times per week for 18 days. Tumor sizes were measured with a caliper. The tumor volume was calculated with the following formula: $v=0.5\left(l \times w^{2}\right)$, where $v$ is volume, $l$ is length, and $w$ is width.

Statistics. Data presented are representative of at least 3 independent experiments. Unless otherwise indicated, data are mean \pm SEM. Statistical significance of differences between means was assessed by a Mann-Whitney test for paired values. Categorical data was analyzed with the $\chi^{2}$ test. All statistical tests were 2 -sided. $P$ values of less than 0.05 were considered to be statistically significant.

\section{Acknowledgments}

We wish to thank H. Bilak for text correction and I. Goddard for excellent support on the mouse study. This work was supported by an institutional grant from CNRS, Centre Léon Bérard (to P. Mehlen), the Ligue Contre le Cancer (to P. Mehlen), ARC (to S. Tauszig-Delamasure), INCA (to P. Mehlen and to J. Bénard), ANR blanche (to P. Mehlen), STREP Hermione (to P. Mehlen), SFCE/Enfants et Santé (to J. Bénard), and Fondation Hubert Gouin (to P. Mehlen). J. Bouzas-Rodriguez is supported by a fellowship from CONACYT, and J.R. Cabrera is supported by the Fondation pour la Recherche Médicale. G. Ichim is supported by the Ligue Contre le Cancer.

Received for publication August 31, 2009, and accepted in revised form January 6, 2010.

Address correspondence to: Patrick Mehlen, Apoptosis, Cancer, and Development Laboratory - Equipe labellisée "La Ligue," CNRS UMR5238, Université de Lyon, Centre Léon Bérard, 69008 Lyon, France. Phone: 33.4.78.78.28.70; Fax: 33.4.78.78.28.87; E-mail:mehlen@lyon.fnclcc.fr.
1. Levi-Montalcini R, Angeletti PU. Essential role of the nerve growth factor in the survival and maintenance of dissociated sensory and sympathetic embryonic nerve cells in vitro. Dev Biol. 1963;7:653-659.

2. Huang EJ, Reichardt LF. Neurotrophins: roles in neuronal development and function. Annu Rev Neurosci. 2001;24:677-736.

3. Nakagawara A. Trk receptor tyrosine kinases: a bridge between cancer and neural development. Cancer Lett. 2001;169(2):107-114.

4. Porter AC, Vaillancourt RR. Tyrosine kinase receptor-activated signal transduction pathways which lead to oncogenesis. Oncogene. 1998;17(11 Reviews):1343-1352.

5. Xu X, Tahan SR, Pasha TL, Zhang PJ. Expression of neurotrophin receptor Trk-C in nevi and melanomas. J Cutan Pathol. 2003;30(5):318-322.

6. Segal RA, Goumnerova LC, Kwon YK, Stiles CD, Pomeroy SL. Expression of the neurotrophin receptor $\mathrm{TrkC}$ is linked to a favorable outcome in medulloblastoma. Proc Natl Acad Sci U S A. 1994;91(26):12867-12871.

7. Grotzer MA, et al. TrkC expression predicts good clinical outcome in primitive neuroectodermal brain tumors. J Clin Oncol. 2000;18(5):1027-1035.

8. Yamashiro DJ, Nakagawara A, Ikegaki N, Liu XG, Brodeur GM. Expression of TrkC in favorable human neuroblastomas. Oncogene. 1996;12(1):37-41.

9. Ryden M, Sehgal R, Dominici C, Schilling FH, Ibanez CF, Kogner P. Expression of mRNA for the neurotrophin receptor trkC in neuroblastomas with favourable tumour stage and good prognosis. Br J Cancer. 1996;74(5):773-779.

10. Brodeur GM, et al. Expression of TrkA, TrkB and TrkC in human neuroblastomas. J Neurooncol. 1997;31(1-2):49-55.

11. Yamashiro DJ, et al. Expression and function of Trk-C in favourable human neuroblastomas. Eur J Cancer. 1997;33(12):2054-2057.

12. Svensson $\mathrm{T}$, et al. Coexpression of mRNA for the full-length neurotrophin receptor trk-C and trk-A in favourable neuroblastoma. Eur J Cancer. 1997;33(12):2058-2063.

13. Kim JY, et al. Activation of neurotrophin-3 receptor TrkC induces apoptosis in medulloblastomas. Cancer Res. 1999;59(3):711-719.

14. Tauszig-Delamasure $S$, et al. The TrkC receptor induces apoptosis when the dependence receptor notion meets the neurotrophin paradigm. Proc Natl Acad Sci U S A. 2007;104(33):13361-13366.

15. Mehlen P, Rabizadeh S, Snipas SJ, Assa-Munt N, Salvesen GS, Bredesen DE. The DCC gene product induces apoptosis by a mechanism requiring receptor proteolysis. Nature. 1998;395(6704):801-804.

16. Mehlen P, Thibert C. Dependence receptors: between life and death. Cell Mol Life Sci. 2004;61(15):1854-1866.

17. Matsunaga E, et al. RGM and its receptor neogenin regulate neuronal survival. Nat Cell Biol. 2004;6(8):749-755. 
18. Bordeaux MC, et al. The RET proto-oncogene induces apoptosis: a novel mechanism for Hirschsprung disease. EMBOJ. 2000;19(15):4056-4063.

19. Furne C, et al. EphrinB3 is an anti-apoptotic ligand that inhibits the dependence receptor functions of EphA4 receptors during adult neurogenesis. Biochim Biophys Acta. 2009;1793(2):231-238.

20. Mourali J, et al. Anaplastic lymphoma kinase is a dependence receptor whose proapoptotic func tions are activated by caspase cleavage. Mol Cell Biol. 2006;26(16):6209-6222.

21. Mehlen P, Bredesen DE. The dependence receptor hypothesis. Apoptosis. 2004;9(1):37-49.

22. Thibert C, Teillet MA, Lapointe F, Mazelin L, Le Douarin NM, Mehlen P. Inhibition of neuroepithelial patched-induced apoptosis by sonic hedgehog. Science. 2003;301(5634):843-846.

23. Furne C, Rama N, Corset V, Chedotal A, Mehlen P. Netrin-1 is a survival factor during commissural neuron navigation. Proc Natl Acad Sci U S A. 2008;105(38):14465-14470.

24. Mehlen P, Puisieux A. Metastasis: a question of life or death. Nat Rev Cancer. 2006;6(6):449-458.

25. Grady WM. Making the case for DCC and UNC5C as tumor-suppressor genes in the colon. Gastroenterology. 2007;133(6):2045-2049.

26. Mazelin L, et al. Netrin-1 controls colorectal tumorigenesis by regulating apoptosis. Nature. 2004;431(7004):80-84.

27. Bernet $A$, et al. Inactivation of the UNC5C Netrin- 1 receptor is associated with tumor progression in colorectal malignancies. Gastroenterology. 2007;133(6):1840-1848.

28. Kinzler KW, Vogelstein B. Lessons from hereditary colorectal cancer. Cell. 1996;87(2):159-170.

29. Maris JM, Hogarty MD, Bagatell R, Cohn SL. Neuroblastoma. Lancet. 2007;369(9579):2106-2120.

30. Stupack DG, et al. Potentiation of neuroblastoma metastasis by loss of caspase-8. Nature. 2006;439(7072):95-99.
31. Delloye-Bourgeois C, et al. Interference with netrin1 and tumor cell death in non-small cell lung cancer. J Natl Cancer Inst. 2009;101(4):237-247.

32. Delloye-Bourgeois C, et al. Netrin-1 acts as a survival factor for aggressive neuroblastoma. J Exp Med. 2009;206(4):833-847.

33. Marchetti D, Nicolson GL. Neurotrophin stimulation of human melanoma cell invasion: selected enhancement of heparanase activity and heparanase degradation of specific heparan sulfate subpopulations. Adv Enzyme Regul. 1997;37:111-134.

34. Innominato PF, Libbrecht L, van den Oord JJ. Expression of neurotrophins and their receptors in pigment cell lesions of the skin. J Pathol. 2001;194(1):95-100.

35. Truzzi F, et al. Neurotrophins and their receptors stimulate melanoma cell proliferation and migration. J Invest Dermatol. 2008;128(8):2031-2040.

36. Calatozzolo C, et al. Expression of cannabinoid receptors and neurotrophins in human gliomas. Neurol Sci. 2007;28(6):304-310.

37. Hisaoka M, Sheng WQ, Tanaka A, Hashimoto H. Gene expression of TrkC (NTRK3) in human soft tissue tumours. J Pathol. 2002;197(5):661-667.

38. Weeraratna AT, Arnold JT, George DJ, DeMarzo A, Isaacs JT. Rational basis for Trk inhibition therapy for prostate cancer. Prostate. 2000;45(2):140-148.

39. Satoh F, et al. Autocrine expression of neurotrophins and their receptors in prostate cancer. Int JUrol. 2001;8(7):S28-34.

40. Miknyoczki SJ, et al. The neurotrophin-trk receptor axes are critical for the growth and progression of human prostatic carcinoma and pancreatic ductal adenocarcinoma xenografts in nude mice. Clin Cancer Res. 2002;8(6):1924-1931.

41. Ketterer K, Rao S, Friess H, Weiss J, Buchler MW, Korc M. Reverse transcription-PCR analysis of laser-captured cells points to potential paracrine and autocrine actions of neurotrophins in pancreatic cancer. Clin Cancer Res. 2003;9(14):5127-5136.
42. Sakamoto Y, et al. Expression of Trk tyrosine kinase receptor is a biologic marker for cell proliferation and perineural invasion of human pancreatic ductal adenocarcinoma. Oncol Rep. 2001;8(3):477-484.

43. Ohta T, et al. Neurotrophin-3 expression in human pancreatic cancers. J Pathol. 1997;181(4):405-412.

44. Du JJ, et al. Expression of NGF family and their receptors in gastric carcinoma: a cDNA microarray study. World J Gastroenterol. 2003;9(7):1431-1434.

45. Ricci A, et al. Neurotrophins and neurotrophin receptors in human lung cancer. Am J Respir Cell Mol Biol. 2001;25(4):439-446.

46. Blasco-Gutierrez MJ, et al. TrkC: a new predictive marker in breast cancer? Cancer Invest. 2007;25(6):405-410.

47. McGregor LM, et al. Roles of trk family neurotrophin receptors in medullary thyroid carcinoma development and progression. Proc Natl Acad Sci U S A. 1999;96(8):4540-4545.

48. Nakagawara A, Brodeur GM. Role of neurotrophins and their receptors in human neuroblastomas: a primary culture study. Eur J Cancer. 1997;33(12):2050-2053.

49. Marchetti D, Nicolson GL. Human melanoma cell invasion: selected neurotrophin enhancement of invasion and heparanase activity. J Investig Dermatol Symp Proc. 1997;2(1):99-105.

50. Sinnappah-Kang ND, Kaiser AJ, Blust BE, Mrak RE, Marchetti D. Heparanase, TrkC and p75NTR: their functional involvement in human medulloblastoma cell invasion. Int J Oncol. 2005;27(3):617-626.

51. Sinnappah-Kang ND, Mrak RE, Paulsen DB, Marchetti D. Heparanase expression and TrkC/ p75NTR ratios in human medulloblastoma. Clin Exp Metastasis. 2006;23(1):55-63.

52. Ghoumari AM, Wehrle R, Bernard O, Sotelo C, Dusart I. Implication of Bcl-2 and Caspase-3 in age-related Purkinje cell death in murine organotypic culture: an in vitro model to study apoptosis. Eur J Neurosci. 2000;12(8):2935-2949. 